PROCEEDINGS OF THE

AMERICAN MATHEMATICAL SOCIETY

Volume 129, Number 8, Pages 2497-2502

S 0002-9939(01)06046-4

Article electronically published on January 18, 2001

\title{
A NOTE ON REGULAR ISOTOPY OF SINGULAR LINKS
}

\author{
CHICHEN M. TSAU
}

(Communicated by Ronald A. Fintushel)

\begin{abstract}
We show that two isotopic oriented 4-valent singular link diagrams with transverse intersections are regularly isotopic if and only if they have the same writhe and the same rotation number.
\end{abstract}

In [13] Whitney (and Graustein) proved that two closed immersed curves in the plane can be homotoped into one another through closed immersed curves if and only if they have the same rotation number. As a generalization of this theorem it is proved in [12] that two isotopic oriented knot diagrams are regularly isotopic if and only if they have the same writhe and rotation number (see also [8, p. 172]). In this note we shall generalize this result further by showing that isotopy of singular link diagrams with transverse intersections can be reduced to regular isotopy if and only if the writhe and rotation number of the corresponding "components" of the singular link diagrams are the same.

An oriented 4-valent singular link $L$ with transverse and flat [14, [11] (or rigid [9]) intersections is the image of a collection of piecewise linear (or piecewise smooth) maps of $S^{1}$ (with the usual parametrization) in $R^{3}$ each with finite (possibly empty) singular set consisting of double points only, and the images of at most two different maps can intersect transversely once at a point which, along with the pair of intersecting arcs, are locally contained in a flat disk.

Isotopy between two 4-valent singular links with transverse and flat intersections is an isotopy that is required to preserve transversality and flatness of intersections at every stage of the isotopy. Regular projection and diagram are defined similar to that of an ordinary oriented link.

Generalized Reidemeister moves (generalized R-moves) for oriented 4-valent singular link diagrams with transverse intersections are the usual three Reidemeister moves for link diagrams together with the following "vertex" moves IV and V [9], 14:

(IV)

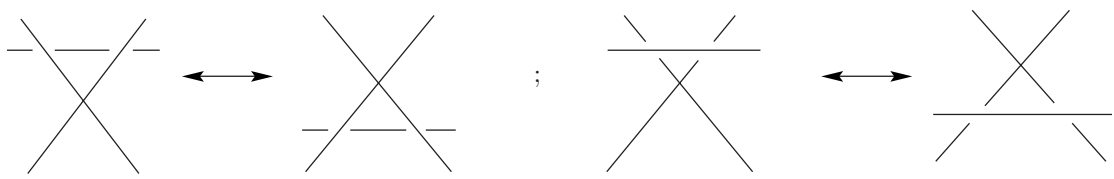

Received by the editors December 13, 1999.

2000 Mathematics Subject Classification. Primary 57M25.

Key words and phrases. Singular link, diagram, generalized Reidemeister moves, writhe, rotation number, regular isotopy. 


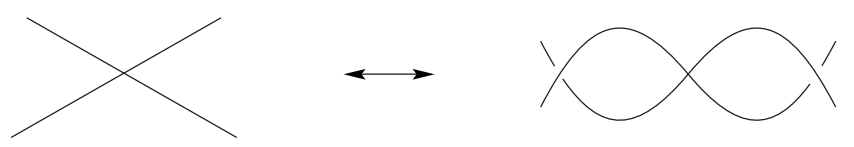

Two oriented singular link diagrams $L_{1}$ and $L_{2}$ with transverse intersections are regularly isotopic if one can obtain a diagram of $L_{2}$ from a diagram of $L_{1}$ (or vice versa) by a sequence of generalized R-moves II, III, IV, and V.

Similar to the Reidemeister Theorem on the isotopy of links in relation to Rmoves I, II, and III, it follows from [9, Theorem 2.1] that two oriented 4-valent singular links with transverse and flat intersections are isotopic if and only if their diagrams are related by a finite sequence of planar isotopies and generalized Rmoves I $\sim \mathrm{V}$.

Define the writhe of an oriented 4-valent singular link diagram $L$ with transverse intersections to be $\operatorname{wr}(L)=\sum_{c} \epsilon(c)$, where the summation is over all the crossings of $L$, ignoring intersections of $L$, and $\epsilon(c)=+1$ or -1 according to whether the crossing $c$ is either $\succ$ or $\succ$. For example, $w r(\ell)=2$. As in the case for oriented link diagrams, $w r$ is a regular isotopy invariant of oriented 4-valent singular link diagrams with transverse intersections. This definition was first given in [10, p. 77] and is purely combinatorial. The author recently uncovered a geometrical explanation and thought it worthy of mentioning here. The geometrical point of view is based on an observation on works of G. Călugăreanu [3], 4] on the double integral (which was later coined the term "writhing number" or "writhe" of $K$ by F. Fuller [5])

$$
\int_{0}^{1} \int_{0}^{1} \frac{(K(u)-K(v)) \cdot K^{\prime}(u) \times K^{\prime}(v)}{\|K(u)-K(v)\|^{3}}
$$

where $K \in C^{\infty}\left([0,1], R^{3}\right)$ is a parametrized smooth knot in $R^{3}$, which he obtained while investigating the behavior in the Gauss integral formula for the linking number of a link of two smooth knots if the knots are allowed to be the same. He showed [3, p. 2] that the double integral $(*)$ always exists as a real number, and (among other things) that [4 p. 614] as one small piece of arc of $K$ is approaching another small piece of arc of $K$ transversely along a fixed direction, then the double integral over the two arcs approaches \pm 1 (the sign depends on the orientation of the arcs involved); and similarly, if for the same pieces of arcs, the direction of approaching is reversed, then the double integral over these two arcs approaches $\mp 1$. Although Călugăreanu mentioned that singularity occurs for the double integral when $K$ has transverse self-intersections, his proof of the above result suggests that the integral $\left(^{*}\right)$ exists when $K$ is a singular knot with transverse intersections. The situation can be described in Figure 1(a), in which $K$ is a singular knot with transverse selfintersections, $w r(K)$ is the double integral $(*)$ (the writhe of $K$ ), and the numbers $w r(K)-1$ and $w r(K)+1$ are obtained by pulling a small piece of arc of $K$, which contains a point of self-intersection of $K$, apart from the other piece of intersecting arc (there are essentially two directions to pull apart), and then approaching the resulting arc to its original position. See Figure 1(b).

Thus one can view $w r(K)$ as the "average" of the writhes of the two approaching (singular) knots, and in the case of oriented knot diagram, the contribution of writhe at a self-intersection would be the average of +1 and -1 , which is 0 .

Recall that the rotation number [13] or rotation index of a closed parametrized curve $C$ immersed in the plane, denoted $r(C)$, is the number of times the unit 


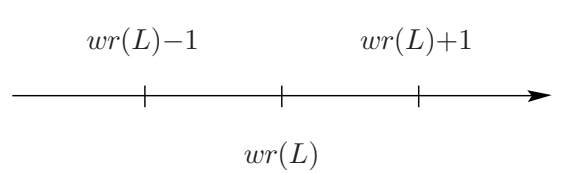

(a)

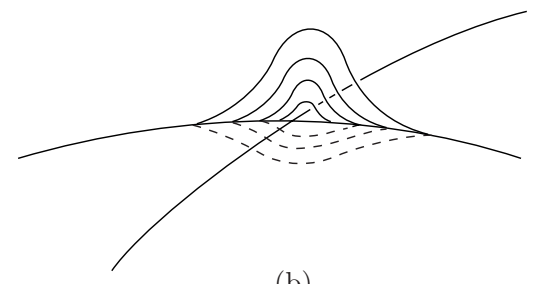

(b)

FIGURE 1.

tangent vector $T(t)=C^{\prime}(t) /\left\|C^{\prime}(t)\right\|$ winds around the (counterclockwise) oriented unit circle $S^{1}$. It is well known that if $C$ is a $C^{2}$-curve, then

$$
r(C)=\frac{1}{2 \pi} \int_{0}^{l} \kappa(s) d s,
$$

the normalized total curvature of $C$. If $C$ is a piecewise linear closed curve with vertices $v_{0}, v_{1}, \ldots, v_{m-1}, v_{m}=v_{0}$, then it is natural to define $r(C)$ to be the normalized total curvature of $C$ as

$$
\frac{1}{2 \pi} \sum_{i=1}^{m} \alpha_{i}
$$

where $\alpha_{i}$ is the signed angle from the vector $v_{i}-v_{i-1}$ to the vector $v_{i+1}-v_{i}$, and $-\pi<\alpha_{i}<\pi$ for all $i$.

One can similarly define the rotation number (or winding number [12, Whitney degree [8] ) for an oriented singular knot diagram $K$, denoted $r(K)$, to be the rotation number of the regular projection for the diagram of $K$. In [8] a combinatorial definition is given for $r(K)$ for any oriented knot diagram $K$. In general, if $L=$ $K_{1} \cup \cdots \cup K_{n}$ is an oriented singular link diagram, then it is natural to define $r(L)=\sum_{i=1}^{n} r\left(K_{i}\right)$. Clearly $r$ is a regular isotopy invariant of oriented 4-valent singular link diagrams with transverse intersections.

It is easy to see that the following local changes of link diagrams are related by planar and regular isotopy (R-moves of types II and III):

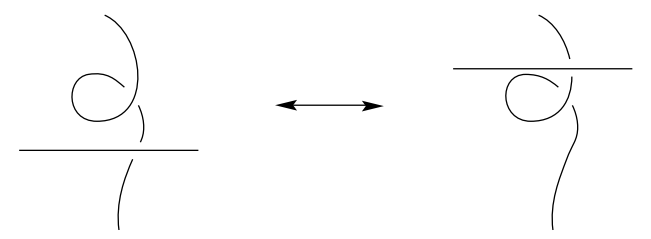

We have a similar result for singular link diagrams:

Lemma. The following local changes of diagrams are related by planar and regular isotopy of oriented singular link diagrams with transverse intersections.

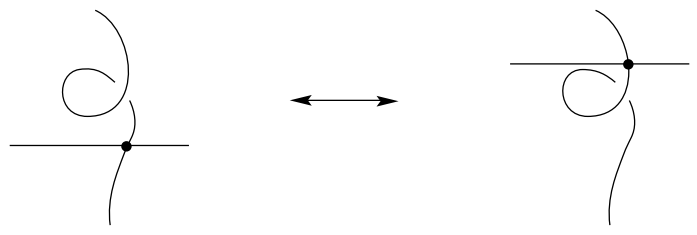


Proof.

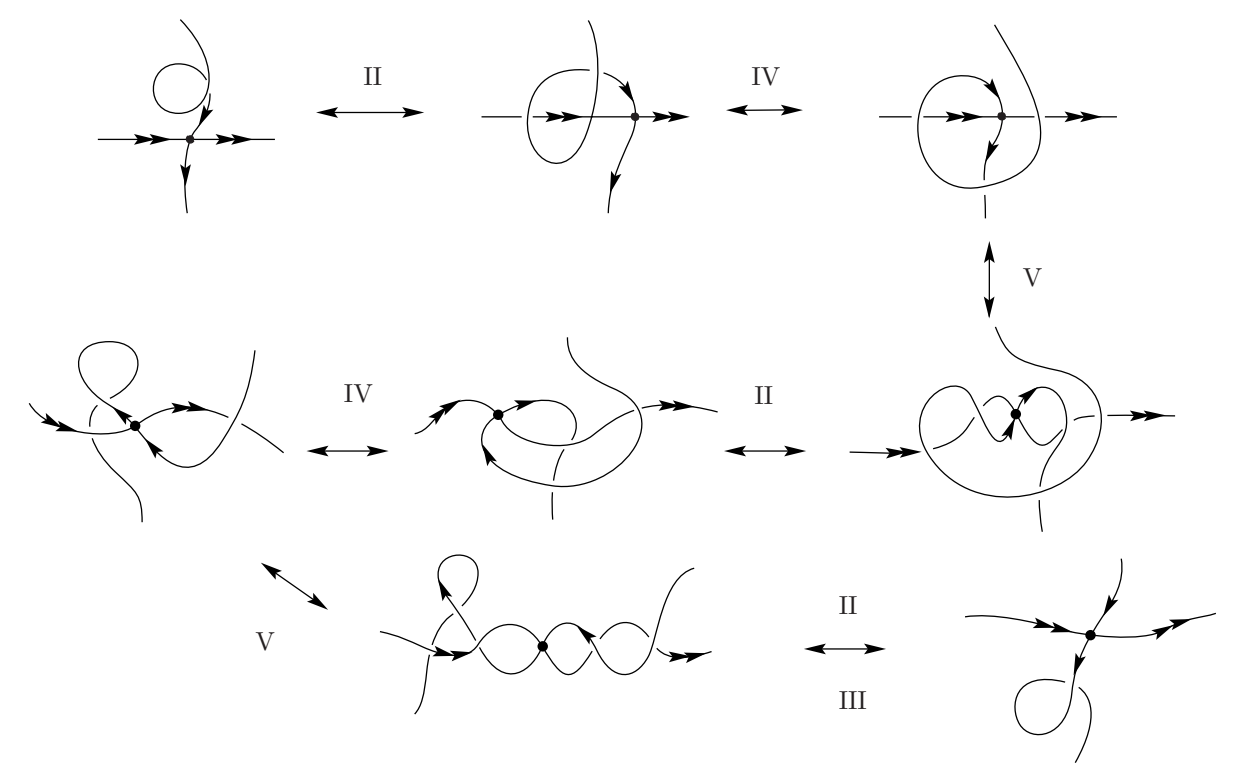

Theorem 1. Let $L_{1}=K_{11} \cup \cdots \cup K_{1 n}$ and $L_{2}=K_{21} \cup \cdots \cup K_{2 n}$ be two isotopic oriented 4-valent singular link diagrams with transverse intersections, and assume the isotopy takes $K_{1 i}$ to $K_{2 i}$ for each $i=1,2, \ldots, n$. Then $L_{1}$ is regularly isotopic to $L_{2}$ where the regular isotopy takes $K_{1 i}$ to $K_{2 i}$ if and only if $w r\left(K_{1 i}\right)=w r\left(K_{2 i}\right)$ and $r\left(K_{1 i}\right)=r\left(K_{2 i}\right)$ for each $i=1,2, \ldots, n$.

Proof. The "only if" part is clear since $K_{1 i}$ is regularly isotopic to $K_{2 i}$ for $i=$ $1,2, \ldots, n$. Conversely, since $L_{1}$ is isotopic to $L_{2}$, a diagram of $L_{2}$ can be obtained from a diagram of $L_{1}$ by a sequence of generalized R-moves I $\sim$ V. Start with $L_{1}$. In dealing with R-moves of type I we use an argument similar to that used in [6], for example. Whenever an R-move of type I is needed to kill a loop, we pull the loop tight to make it very small, and then carry it along all successive moves as if it weren't there at all, promising to pay attention to it at the end of the procedure. If an R-move of type I is needed to give birth to a loop, we create a pair of opposite loops as shown in the following figures:

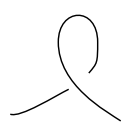

Need

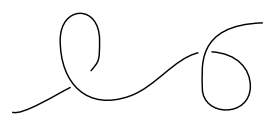

Create

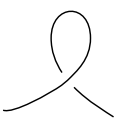

Need

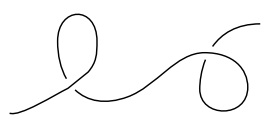

Create

Note that each pair of opposite loops is regularly isotopic to a trivial arc (see, for example, [8 p. 172]). We then pull one of the loops, which is not needed, very tight to make it very small, and then proceed as before. Using Lemma 6 , we can get together, via regular isotopy, those tiny loops on each $K_{1 i}$, which remain as souvenirs of R-moves of type I, into a portion of $K_{1 i}$ that is contained in a small disk $\Delta$ separated from the rest of the link. Let $L_{1}^{\prime}=\bigcup_{i=1}^{n} K_{1 i}^{\prime}$ be the singular 
link diagram obtained at the end of the sequence of moves applied on $L_{1}$ described above. Then $L_{1}^{\prime}$ is regularly isotopic to $L_{1}$, and the link diagrams $L_{1}^{\prime}$ and $L_{2}$ are exactly the same except on each $K_{1 i}^{\prime}$ there may be a chain of type I loops which are extra comparing to $K_{2 i}$. Now since $w r(\ell \sigma)=r(\varrho \sigma)=0$, no matter what orientation of the arc is, it follows that $w r\left(K_{1 i^{\prime}}\right)=w r\left(K_{1 i}\right)$ and $r\left(K_{1 i^{\prime}}\right)=r\left(K_{1 i}\right)$ for each $i=1,2, \ldots, n$, and hence $w r\left(K_{1 i^{\prime}}\right)=w r\left(K_{2 i}\right)$ and $r\left(K_{1 i^{\prime}}\right)=r\left(K_{2 i}\right)$ for each $i=1,2, \ldots, n$. Therefore $L_{2}$ is regularly isotopic to $L_{1}^{\prime}$ by [12] (see also [8] Proposition, p. 172]), and the regular isotopy can take place inside the disk $\Delta$. Combining the regular isotopies from $L_{1}$ to $L_{1}^{\prime}$ and from $L_{1}^{\prime}$ to $L_{2}$ gives the required regular isotopy from $L_{1}$ to $L_{2}$.

Remark. Since for any oriented link diagram $L=K_{1} \cup \cdots \cup K_{n}, w r(L)=2 l k(L)+$ $\sum_{i=1}^{n} w r\left(K_{i}\right)$, where $l k(L)=\sum_{1 \leq i<j \leq n}^{n} l k\left(K_{i}, K_{j}\right)$, it follows that if $L_{1}$ is isotopic to $L_{2}$, then $\left(l k\left(L_{1}\right)=l k\left(L_{2}\right)\right.$ and $) \operatorname{wr}\left(L_{1}\right)=w r\left(L_{2}\right)$ if and only if $\sum_{i=1}^{n} \operatorname{wr}\left(K_{1 i}\right)=$ $\sum_{i=1}^{n} w r\left(K_{2 i}\right)$. Thus the "if" condition in the Theorem above implies (but is not equivalent to) $\operatorname{wr}\left(L_{1}\right)=w r\left(L_{2}\right)$ and $r\left(L_{1}\right)=r\left(L_{2}\right)$. Note also that the converse of the fact that if $L_{1}$ is regularly isotopic to $L_{2}$, then $\operatorname{wr}\left(L_{1}\right)=\operatorname{wr}\left(L_{2}\right)$ and $r\left(L_{1}\right)=$ $r\left(L_{2}\right)$ is not true; for example, $w r(8 \&)=w r(\bigcirc \bigcirc)=0$ and $r(8 \&)=r(\bigcirc \bigcirc)=0$, but these two links are not regularly isotopic.

The following gives a straightforward generalization of the Mirror Theorem in [8, p. 173].

Corollary 2. Let $L=K_{1} \cup \cdots \cup K_{n}$ be an oriented 4-valent singular link diagram with transverse intersections, and assume $w r\left(K_{i}\right)=0$ for each $i=1,2, \ldots, n$. Then $L$ is isotopic to its mirror image $\bar{L}$ if and only if $L$ is regularly isotopic to $\bar{L}$.

Proof. Use the above theorem and the fact that $\operatorname{wr}(L)=-w r(\bar{L})$ and $r(L)=$ $r(\bar{L})$.

The following gives a similar result for invertible singular link diagrams:

Corollary 3. Let $L=K_{1} \cup \cdots \cup K_{n}$ be an oriented 4-valent singular link diagram with transverse intersections, and assume $r\left(K_{i}\right)=0$ for each $i=1,2, \ldots, n$. Then $L$ is isotopic to to its inverse $L^{-1}$ if and only if $L$ is regularly isotopic to $L^{-1}$.

Proof. This follows from the above theorem and the fact that $w r(L)=w r\left(L^{-1}\right)$ and $r(L)=-r\left(L^{-1}\right)$.

Remark. Since regular isotopy of singular link diagrams preserves writhe, a 3dimensional version of Theorem 1 in the case of oriented knot diagram is given in 1], 7], and [2, which states that if two parametrized knots are isotopic and have the same writhing number, then they can be isotoped to each other through knots with the same writhing number.

\section{ACKNOWLEDGEMENT}

The author would like to thank the referee for pointing out a mislabeled diagram in the original manuscript and for helpful comments. The author would also like to take this opportunity to thank the Institute of Mathematics of the Academia Sinica in Taiwan, Republic of China, for providing support during his visit at the Institute. Thanks also due to Jim Hebda for helpful conversations. 


\section{REFERENCES}

1. C. Benham and D. Miller, Fixed-writhe isotopies and the topological consevation law for closed, circular DNA, J. of Knot Theory and Its Ramifications 5 (1996), 859-866. MR 98b:57019

2. C. Benham, X.-S. Lin and D. Miller, Subspaces of knot spaces, preprint.

3. G. Călugăreanu, L'intégrale de Gauss et l'analyses des naeuds tridimensionnels, Rev. Math. pures appl. 4 (1959), 5-20. MR 24:A1693

4. G. Călugăreanu, Sur les classes d'isotopie des noeuds tridimensionnels et leurs invariants, Czechoslovak Math. J. 11 (1961), 588-625. MR 24:A2351

5. F. Fuller, The writhing number of a space curve, Proc. Nat. Acad. Sci. USA 68 (1971). MR 43:3928

6. H. Gluck and L-H. Pan, Knot Theory in the presence of curvature, preprint.

7. J. Hebda and C. M. Tsau, Normal holonomy and Writhing number of polygonal knots, preprint.

8. L. Kauffman, On Knots, Annals of Math. Studies, no. 115, Princeton Univ. Press, 1987. MR 89c:57005

9. L. Kauffman, Invariants of graphs in 3-space, Trans. Amer. Math. Soc. vol. 311, no. 9 (1989), 417-465. MR 89f:57007

10. L. Kauffman and P. Vogel, Link polynomial and a graphical calculus, J. of Knot Theory and Its Ramifications vol. 1, no. 1 (1992). MR 92m:57012

11. K. Murasugi, Knot Theory, Birkhäuser, 1996. MR 97g:57011.

12. B. Trace, On the Reidemeister moves of a classical knot, Proc. Amer. Math. Soc. vol. 89, no. 4 (1983), 722-724. MR 85f:57005

13. H. Whitney, On regular closed curves in the plane, Compositio Math. 4 (1937), 276-284.

14. S. Yamada, An invariants of spatial graphs, J. of Graph Theory vol. 13, no. 5 (1989), 537-551. MR 90j:57004

Department of Mathematics and Mathematical Computer Science, Saint Louis UniVersity, St. Louis, Missouri 63103

E-mail address: tsaumc@slu.edu 